\title{
Correction to: Long-Term RST Analysis of Anomalous TIR Sequences in Relation with Earthquakes Occurred in Greece in the Period 2004-2013
}

\author{
Alexander Eleftheriou, ${ }^{1}$ Carolina Filizzola,${ }^{2}$ Nicola Genzano, ${ }^{3}$ Teodosio Lacava, ${ }^{2}$ Mariano Lisi, ${ }^{3}$ \\ Rossana Paciello, ${ }^{2}$ Nicola Pergola, ${ }^{2,3}$ Filippos Vallianatos, ${ }^{1}$ and Valerio Tramutoli ${ }^{2,3,4}$
}

Correction to: Pure Appl. Geophys. 173 (2016), 285-303 https://doi.org/10.1007/s00024-015-1116-8

1. Unfortunately, Fig. 8 has been published incorrect. Please find the corrected Fig. 8 below:
2. Due to an unfortunate oversight the sentence “... A probability gain (compared with a random guess) from 1.5 up to 3.7 was achieved as far as only SSTAs preceding earthquakes with $\mathrm{M} \geq 5.5$ are considered. ..." is not given correctly.

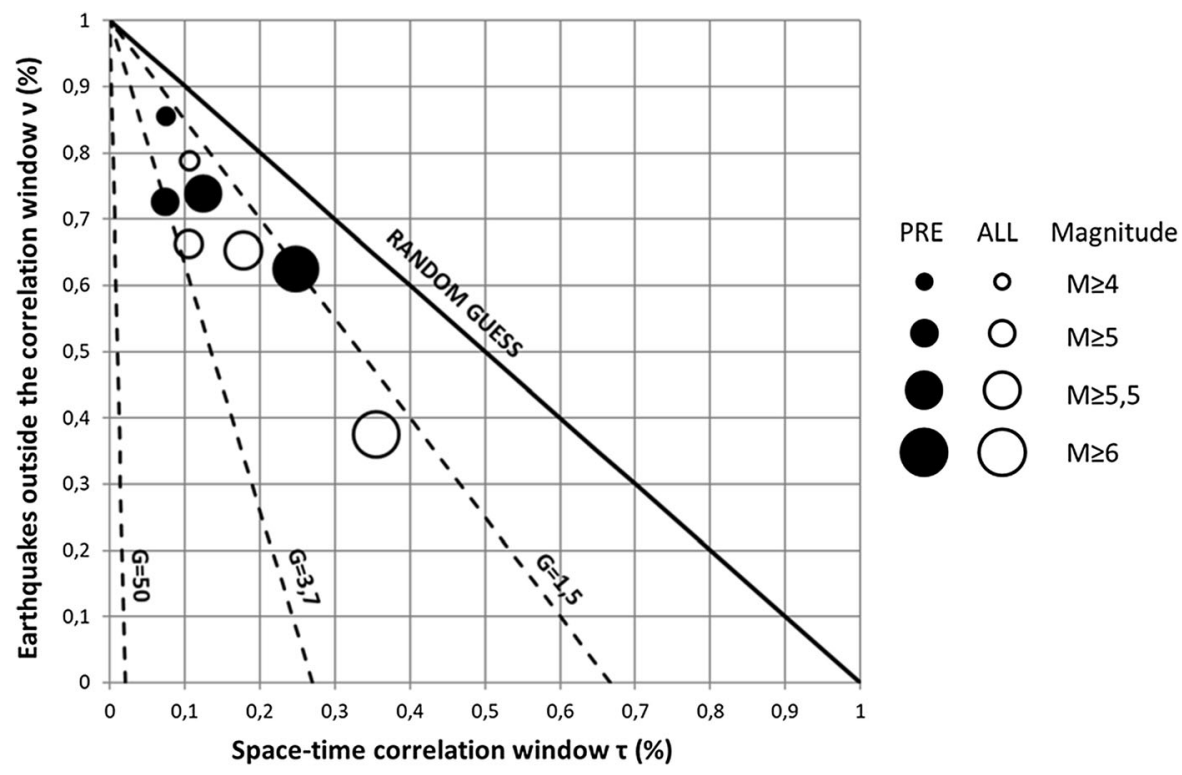

The original article can be found online at https://doi.org/10.1007/ s00024-015-1116-8.

1 Department of Environmental and Natural Resources Engineering, School of Applied Sciences, Technological Educational Institute of Crete, Chania, Crete.

2 Institute of Methodologies for Environmental Analysis of the National Research Council, Tito Scalo, PZ, Italy.

3 School of Engineering, University of Basilicata, Via dell'Ateneo Lucano 10, 85100 Potenza, Italy.

4 International Space Science Institute, Bern, Switzerland.
It should read: “... A probability gain (compared with a random guess) from 1.5 up to 3.7 was achieved as far as only SSTAs preceding earthquakes with $\mathrm{M} \geq 5$ are considered. ...".

The authors apologize for this mistake.

The original article was updated. 